\title{
Impurity survey after Boronization in ASDEX Upgrade measured by a collector probe
}

\author{
W. Schustereder ${ }^{*}$, A. Herrmann, V. Rohde, ASDEX Upgrade Team \\ Max-Planck-Institut für Plasmaphysik, EURATOM Association, 85748 Garching, Germany
}

\begin{abstract}
Wall conditioning is carried out in today's fusion machines to control the plasma impurity content. In ASDEX Upgrade boronization is routinely used to optimize the plasma start-up in the limiter phase and the performance of low density, high power scenarios. We present data on the impurity content in the scrape-off layer (SOL) immediately after a boronization monitored at eight consecutive, nearly identical H-mode discharges. Dischargeresolved measurements of the deposition of impurity species on a collector probe were carried out in the outer midplane of ASDEX Upgrade. While the amount of collected boron decreases slowly the impurity concentration of most elements increases slowly and steadily. The collected amount of the $1^{\text {st }}$ wall material tungsten was below the detection limit. Spectroscopic data observe an increase at very low level. In contrast $\mathrm{Ca}$ and $\mathrm{Fe}$, possible precursors of dust formation, increase at a significantly higher rate.
\end{abstract}

PACS: $28.52 . \mathrm{Fa}, 52.25 . \mathrm{Vy}, 52.40 . \mathrm{Hf}$

Corresponding Author Address: Dr. Werner Schustereder, Boltzmannstr. 2, 85748 Garching, Germany, Phone: + 4989 - 3299 1468, Fax: + 4989 - 32991212

Corresponding Author e-mail: Werner.Schustereder@ipp.mpg.de 


\section{Introduction}

In 2007 ASDEX Upgrade will turn into a full tungsten machine. For the initial start-up a fresh boronization with $10 \% \mathrm{~B}_{2} \mathrm{H}_{6}$ and $90 \% \mathrm{He}$ will be applied. Boronization is long known to improve various substantial parameters crucial for the operation in advanced conditions. In contrast to other conditioning techniques (e.g. carbonization) boronization does not pose any problem with density control. The density limit and the plasma conditions are durably improved after boronization. The effective particle confinement is high, as is typical of metallic walls. High-Z impurities are depressed efficiently, also the concentrations of light impurities like carbon as well as volatile compounds as oxygen and $\mathrm{H}_{2} \mathrm{O}$ are lowered considerably. The oxygen-diminishing effect of boronization remains over a long period (hundreds of discharges) and older layers can be partly reactivated by plasma discharges even after venting of the vessel and maintenance work inside it [1].

Boron layers are resistant to chemical erosion. Since boron is removed by sputtering and the sputtering yield being strongly dependent on the nature of the incident particles it is crucial to measure the amount and composition of the plasma impurities impinging on the boronized first wall. We present an impurity survey recorded with a deposition probe exposed to the midplane scrape-off layer (SOL) of ASDEX Upgrade immediately after boronization. Eight nearly identical, consecutive $\mathrm{H}$-mode discharges are monitored and the impurity content is measured. This will contribute to the understanding of the initial start-up of the full tungsten ASDEX Upgrade in 2007.

\section{Experimental}

The exposed sample is a cylinder made of pyrolytic graphite with a length of $88 \mathrm{~mm}$ and a diameter of $30 \mathrm{~mm}$ (Fig. 1b). It is exposed to discharges using the midplane manipulator system of ASDEX Upgrade (as shown in Fig. 1 of [2]), located at $\mathrm{z}=0.31 \mathrm{~m}$ above the midplane in the shadow of an ICRH limiter and near a protection limiter. To obtain a well 
defined plasma wetted surface, the samples were mounted inside a graphite shield with a narrow slit aperture defining a viewing cone of $12^{\circ}$ (Fig. 1a). The slit is oriented parallel to the sample in radial direction. To obtain discharge-resolved measurements the cylindrical probe is rotated by an angle of $30^{\circ}$ inside the shield between the discharges. The width of the gap between the probe surface and the inside of the shield is $1.5 \mathrm{~mm}$. This is considerably larger than the Larmor radius of deuterium ions in the far SOL which is about $0.6 \mathrm{~mm}$ for an expected maximum ion energy of about $50 \mathrm{eV}\left(\mathrm{T}_{\mathrm{e}} \leq 20 \mathrm{eV}[3]\right)$. Impurity ion species, however, have due to their larger mass/charge ratio a larger Larmor radius that can be in the order of millimeters, thereby potentially causing a broadening of the deposition stripes on the collector probe behind the slit aperture.

Exposure was done immediately after boronization in eight nearly identical, consecutive $\mathrm{H}$ mode discharges (\#21404-\#21411). These stationary discharges in lower single null configuration drew a current of $\mathrm{I}_{\mathrm{P}}=1 \mathrm{MA}$ in a toroidal field of $\mathrm{B}_{t}=-2.3 \mathrm{~T}$ and a line averaged density of $n_{e}=7.3 \times 10^{19} \mathrm{~m}^{-3}$. While the safety factor $\mathrm{q}_{95}=4.2$ and the neutral beam heating power $\mathrm{P}_{\mathrm{NBI}}=5.2 \mathrm{MW}$ are kept constant, the discharges 5-7 (\#21408-\#21410) are additionally heated with ion cyclotron resonance heating $\mathrm{P}_{\mathrm{ICRH}}=3.0 \mathrm{MW}$ from $\mathrm{t}=5.5-6.5 \mathrm{~s}$. At the end of the discharges 6 - 8 (\#21409-\#21411) a fast forward gas feed is applied.

After exposure the retrieved probe was analyzed ex-situ using Rutherford back-scattering (RBS), Nuclear Reaction Analysis (NRA) and Proton Induced X-ray Emission (PIXE).

Rutherford back-scattering (RBS) spectra were taken employing ${ }^{3} \mathrm{He}^{+}$ions at $0.8 \mathrm{MeV}$ with perpendicular impact of the projectiles on the sample. Recoil ions were detected at a scattering angle of $165^{\circ}$. An ion charge of $10 \mu \mathrm{C}$ in the case of ${ }^{3} \mathrm{He}^{+}$was typically acquired for each spectrum. The resolution for heavy elements like Fe and $\mathrm{W}$ is generally good, allowing accurate quantification of areal densities of these plasma impurities on the sample. SIMNRA 5.84 [4] was used as source of cross section data and as a tool for simulation and 
fitting of the RBS spectra. Signals of mid- and low-Z materials are often overlapping with those of other elements, making quantitative statements difficult.

Therefore the amount of mid- and low-Z trace elements deposited onto the samples was additionally quantified by particle-induced X-ray emission (PIXE) using a $1.5 \mathrm{MeV}$ proton beam. The detection of characteristic X-ray lines from all elements heavier than F allowed quantitative analysis using cross section data published by H. Paul and J. Sacher [5]. Due to the sharp line width signals are usually nearly free from interference with characteristic lines from other chemical elements. Compared with RBS, this technique has in general a lower sensitivity but a higher resolution.

The near-surface amounts of deuterium were measured by nuclear reaction analysis (NRA) using the reaction ${ }^{3} \mathrm{He}(\mathrm{d}, \mathrm{p}) \alpha$. The analyzing depth with the employed incident ${ }^{3} \mathrm{He}^{+}$energy of $800 \mathrm{keV}$ is in the range of $1 \mu \mathrm{m}$. Quantification is achieved by comparison to reference samples with a-C:D layers of known thickness and composition.

\section{Results}

It is clear that discharge-integrated exposures of the collector sample show a clear correlation of the impurity amount deposited at the probe with the radial distance $d_{S P}$ of the separatrix to the probe. Therefore we kept $d_{S P}=60 \mathrm{~mm}$ constant for all eight, consecutive discharges to avoid additional effects.

Along with the deuterium and boron content various impurity species were recorded. Surprisingly they can be divided into three groups, showing different behavior.

\subsection{Discharge resolved measurement of deuterium, $B$ and $W$}

The deposition of deuterium on the collector probe is shown in Fig. 1 and nicely resolves the eight consecutive discharges. After each deposition the sample was turned approximately $30^{\circ}$. The amount of collected boron decreases slowly as expected. Due to the fresh layer of boron 
on the $1^{\text {st }}$ wall tungsten is covered and was not found on the collector sample in amounts detectable with ion beam analysis (IBA) techniques. Therefore time integrated spectroscopic data with a line of sight towards the IRCH limiter near the collector probe are presented observing a slow increase of tungsten.

\subsection{Impurities rising slowly and constantly}

Most impurities show a quasi-linear increase with the number of discharges after boronization, see Fig. 2. At the $5^{\text {th }}$ and $6^{\text {th }}$ discharge, the first discharges with short, temporal ICR heating and fast forward gas feed, an additional increase of about $25-75 \%$ of the impurity content is measured. From the $6^{\text {th }}$ discharge on the impurity content levels at about constant values. Comparing the absolute amount of these impurity species after the $8^{\text {th }}$ discharge to 'normal' operation conditions (not directly after boronization) we see a difference of a factor of $10-20$, a clear effect of the boronization.

\subsection{Impurities rising slowly, but showing a maximum concentration in discharge 6}

Another group of impurity species shows a rather unclear behaviour. The concentration found on the deposition sample is nearly constant for all eight discharges, except for a pronounced peaking at the $6^{\text {th }}$ discharge, being the first discharge with simultaneous ICR heating and fast forward gas feed at the end of the discharge. The values in this discharge are about $200-$ $300 \%$ higher than before and after this discharge that shows no differences to the identical $7^{\text {th }}$ discharge. In this case one can think of a local source not necessarily causally related to the actual discharge. After the $8^{\text {th }}$ discharge the absolute impurity amount is again about a factor of $10-20$ lower than in normal operation conditions.

\subsection{Strongly increasing impurities}


In contrast to all other impurity species $\mathrm{Ca}$ and Fe increase steadily from the $1^{\text {st }}$ discharge on. The slope rises during the $5^{\text {th }}$ discharge and $6^{\text {th }}$ discharge, while the $8^{\text {th }}$ discharge shows the same amount of $\mathrm{Fe}$ and $\mathrm{Ca}$ as the $7^{\text {th }}$. This indicates an influence of the additionally employed ICR heating during these discharges, which is on the one hand in agreement with previous studies proving the influence of ICR heating, on the other hand raises the question whether a one second period of heating should be sufficient for efficient removal of the boron layer. This is more than questionable, other impurity species described above do not show such a strong increase. A possible explanation is that these most abundant impurity species do not origin from the $1^{\text {st }}$ wall but from the isolation of electrical cables. Interestingly $\mathrm{Ca}$ and $\mathrm{Fe}$ are the dominant elements in the isolation material and the outer covering of electric cables and are also expected to be the precursors of dust formation in AUG [2]. These cables are likely to not being well-covered by boronization. They are moving slightly during discharges, resulting in development of cracks. Release of material by the cracks may lead to the formation of dust $[6,7]$ which migrates through the machine and is subsequently redeposited at various positions - also at the midplane manipulator probe.

In contrast to the other impurity species $\mathrm{Ca}$ and $\mathrm{Fe}$ are after the $8^{\text {th }}$ discharge only factors of $3-4$ lower than in normal operation conditions.

\subsection{Observations valid for all impurity species}

At each angular position of the sample one can perform also radial scans of the deuterium and impurity content. Taking the exponential part of a radial scan we calculate the radial fall-off length of each impurity in the limiter shadow. This indicates if the collected impurity species diffuses from the hot core plasma or if it is mainly transported via edge plasma flows. In the first case the radial fall-off length allows to estimate the impurity concentration at the limiter edge. This applies for all presented impurity species with a radial fall-off length of typically 2-4 mm. 
Overall it can be observed that all impurity species show broad angular distributions as compared to the pronounced peaking illustrated for deuterium. It is expected that along with the gyro-motion of the incoming ions also sputtering leads to a broadening of the peaks. It is currently under investigation how this affects the properties of the deposition probe and its shielding with special regard to the determination of the absolute amount of collected impurity species, which seems an especially important issue for $\mathrm{Ca}$ and $\mathrm{Fe}$.

\section{Discussion and Outlook}

ASDEX Upgrade will turn into a full tungsten machine in 2007. For the initial start-up a boronization will be applied. Here we showed by measurements with a deposition probe that the impurity content immediately after boronization is considerably lower than at normal operation with metal first walls. Most impurity species rise slowly in concentration during the first eight discharges. In contrast a strong increase for Fe and $\mathrm{Ca}$ is observed. These elements are expected to origin from the isolation of electric cables and are possible precursors for dust formation. We will try to verify these findings by an impurity survey at the beginning of the 2007 campaign of ASDEX Upgrade. We will in parallel monitor the degradation of the boron layer at start-up and several times thereafter, as well as the amount of residual carbon. Furthermore erosion measurements of various materials will yield a quantification of the average ion energy at the midplane. Erosion and redeposition will be investigated using marker probes along with laser blow-off injectory experiments. A fast stroke into the outer core plasma is envisaged. Great attention will be placed on studying the processes leading to dust formation and its precursors.

\section{Acknowledgements}

We acknowledge the help of the staff at the section for material analysis and the engineers responsible for the ASDEX Upgrade midplane manipulator system. 


\section{References}

[1] Schneider U. et al. 1990 J.Nucl.Mat. 176 \& 177350

[2] Schustereder W. et al 2006 Discharge resolved impurity flux measurements in the edge plasma of ASDEX Upgrade by exposure of collector probes J.Nucl.Mat. at press

[3] Neuhauser J. at al. 2002 Plasma Phys. Control. Fusion 44855

[4] Mayer M., SIMNRA User's Guide, Report IPP 9/113, Max-Planck-Institut für Plasmaphysik, Garching, Germany, 1997

[5] Paul H. and Sacher J. 1989 Atomic Data and Nuclear Data Tables 42105

[6] Winter J. and Gebauer G. 1999 J. Nucl. Mat. 266-269 228

[7] J.P. Sharpe et al. 2002 Fusion Engineering and Design 63-64 153 


\section{Figure captions}

Fig. 1: Heat resistant graphite shielding (a) for protection of the manipulator probe (b) at the midplane manipulator system of ASDEX Upgrade. The probe is situated inside the shielding, deposition stripes are obtained by rotating the probe between the discharges.

Fig. 2: The deposition of deuterium on the collector probe nicely resolves the eight consecutive discharges. The amount of collected boron decreases slowly. Spectroscopic data observe an increase of $\mathrm{W}$ at very low level, below the detection limit of IBA techniques.

Fig. 3: Discharge-resolved signals of $\mathrm{Ti}, \mathrm{V}, \mathrm{Cr}$ and $\mathrm{Mn} \mathrm{K}_{\mathrm{alpha}}$ as well as $\mathrm{Zr} \mathrm{L}_{\text {alpha }}$ lines converted into areal densities at the deposition sample.

Fig. 4: Discharge-resolved signals of $\mathrm{Cl}$ and $\mathrm{K} \mathrm{K}_{\text {alpha }}$ as well as $\mathrm{Mo} \mathrm{L}_{\text {alpha }}$ lines converted into areal densities at the deposition sample.

Fig. 5: $\mathrm{Ca}$ and $\mathrm{Fe}$ show a steep increase from the $5^{\text {th }}$ discharge on, levelling after the $7^{\text {th }}$ discharge. These are the discharges with short additional ICR heating. Interestingly $\mathrm{Ca}$ and $\mathrm{Fe}$ are the main components of the isolation of electric cables in ASDEX Upgrade and possible precursors of dust formation. 
Figures

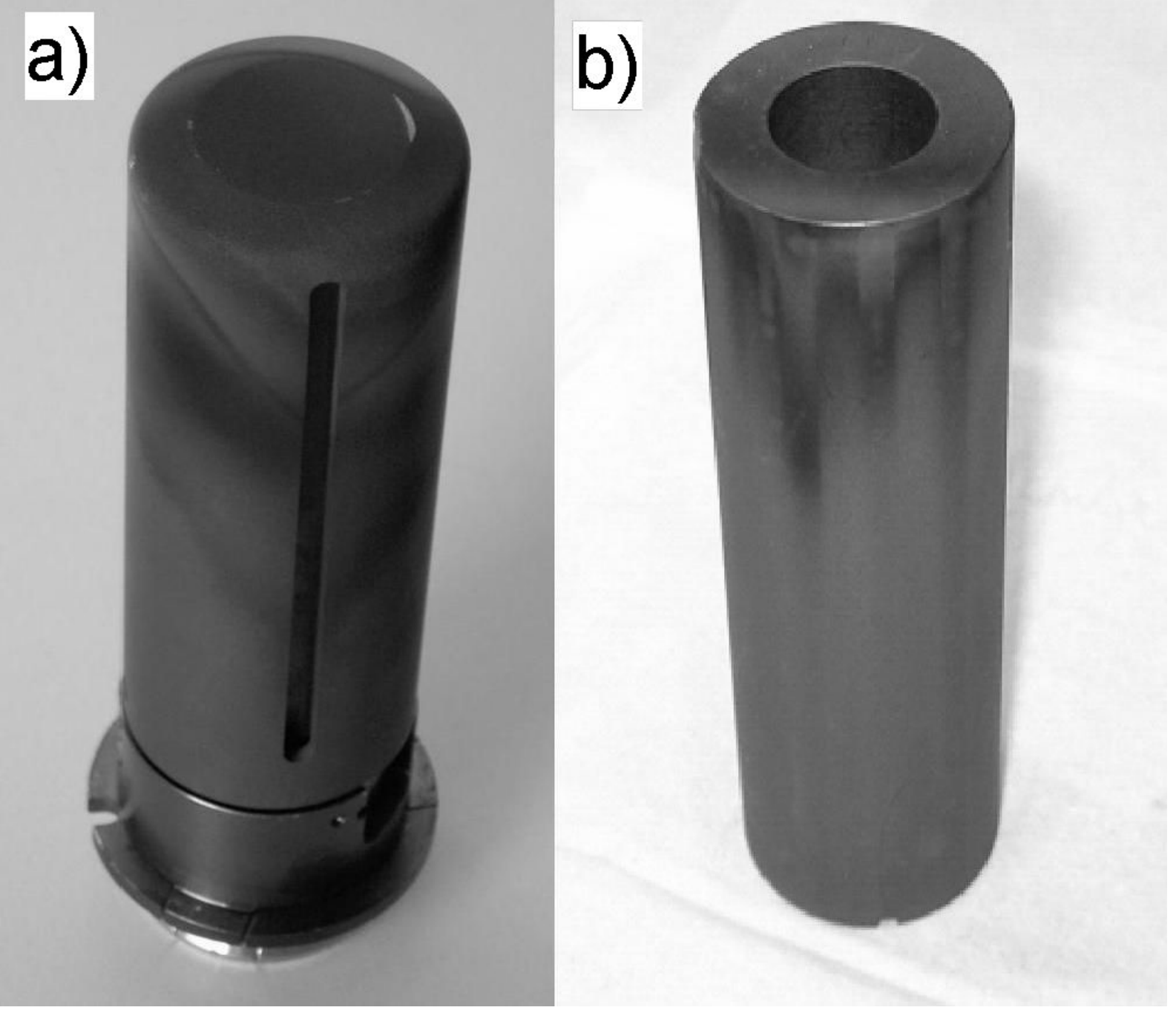

Fig. 1 


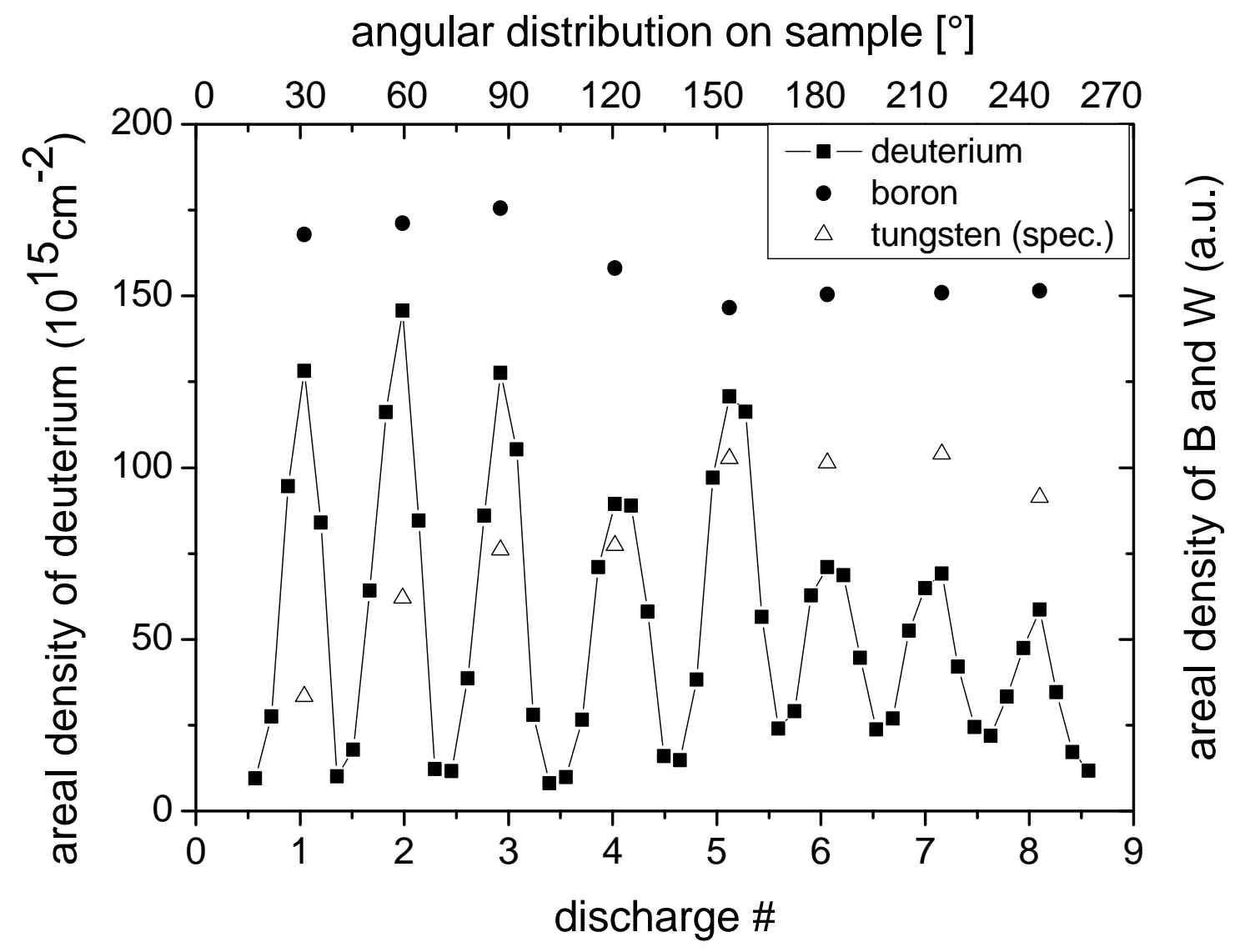

Fig. 2 


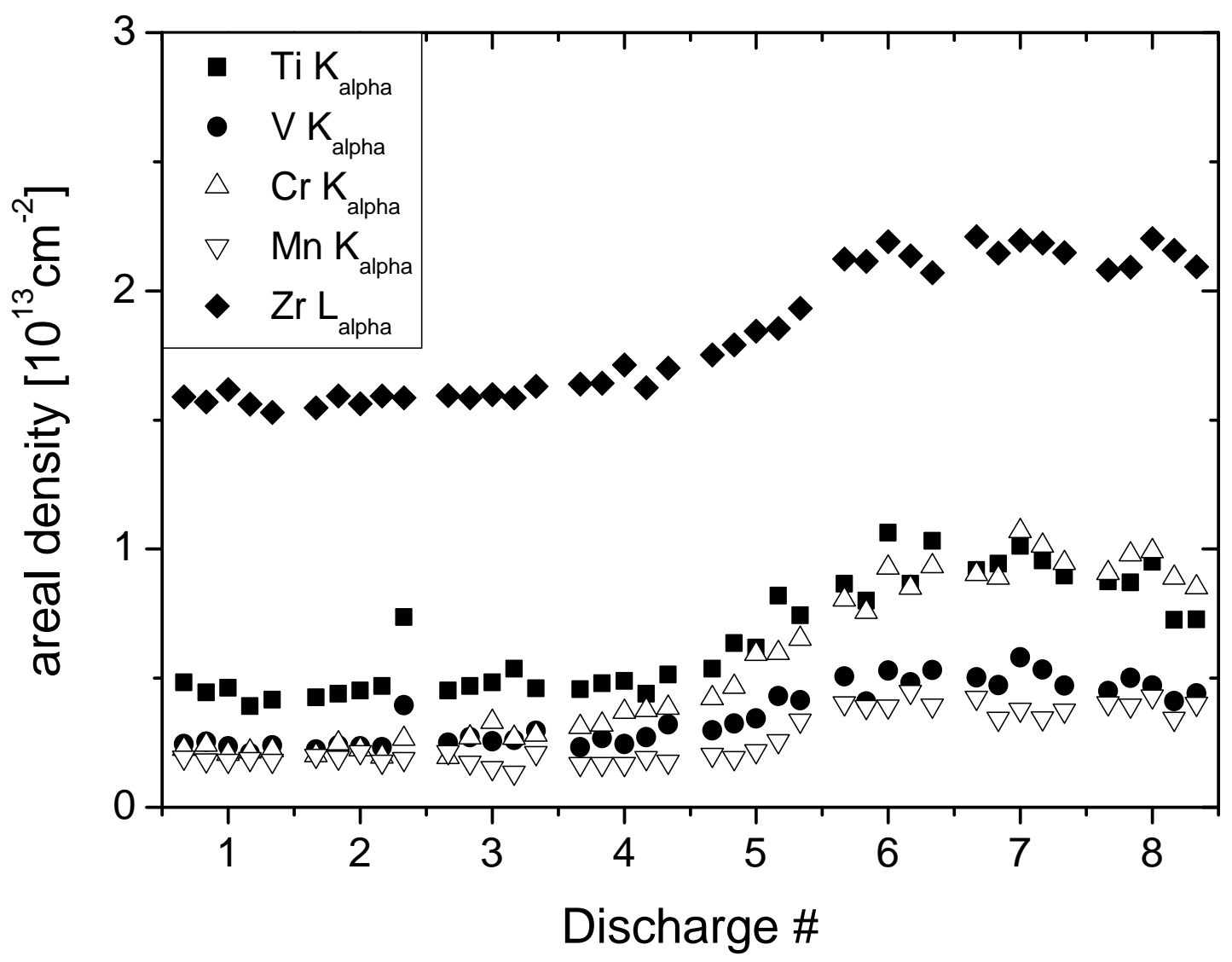

Fig. 3 


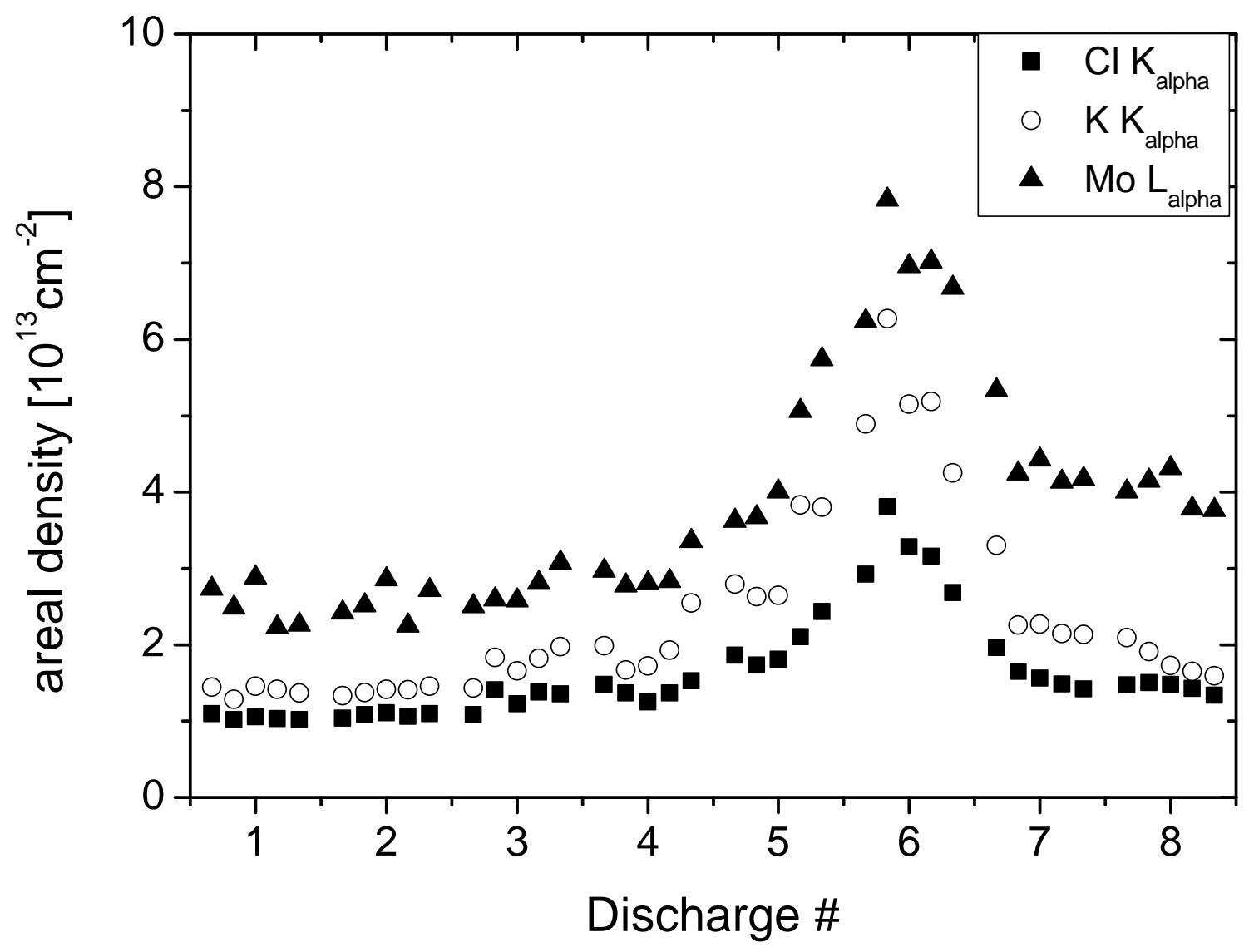

Fig. 4 


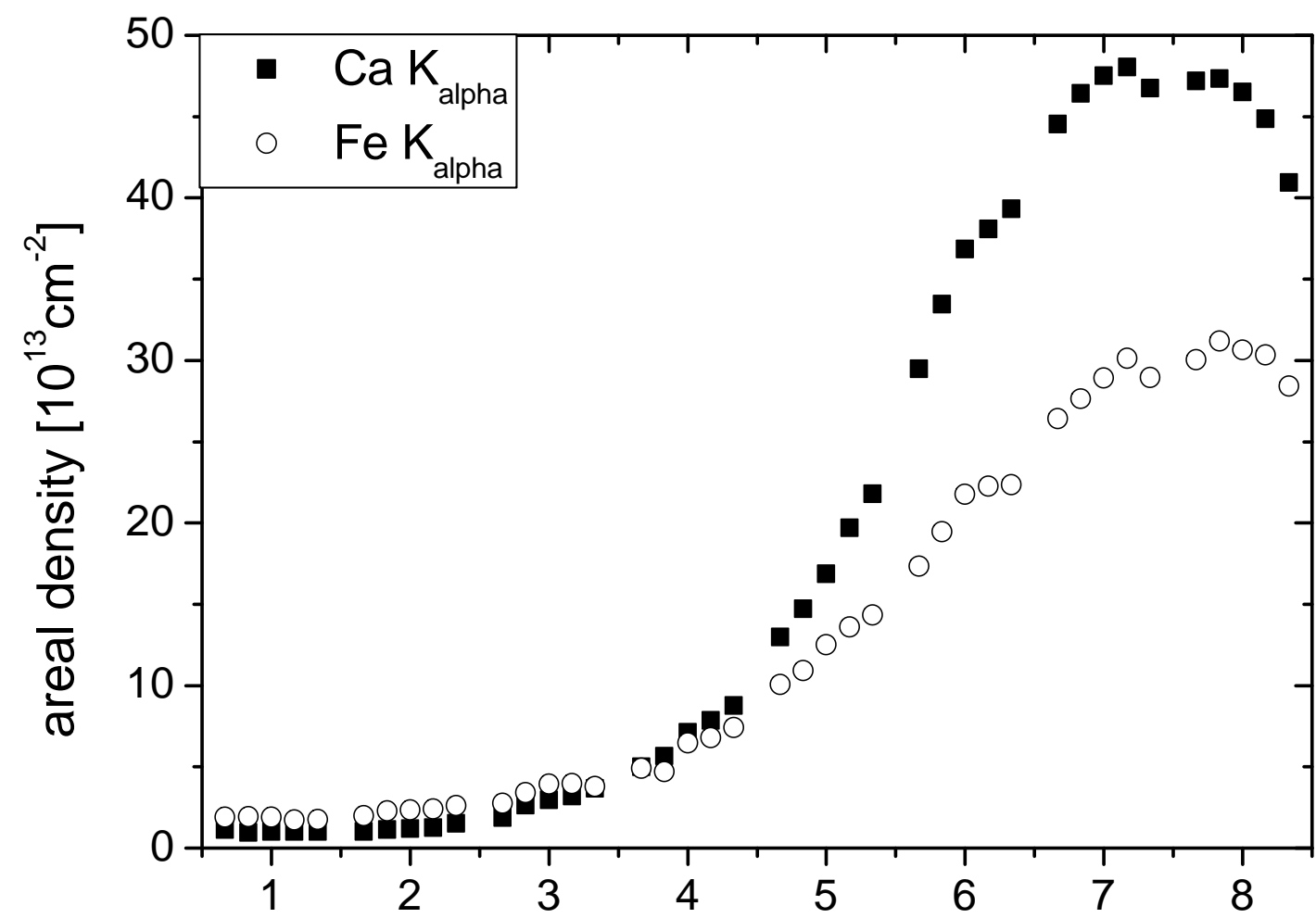

Discharge \#

Fig. 5 